\title{
STABILITY AND STABILISATION OF 2D DISCRETE LINEAR SYSTEMS WITH MULTIPLE DELAYS
}

\author{
Wojciech Paszke ${ }^{1}$, Krzysztof Gatkowski ${ }^{1}$, James Lam ${ }^{2}$, Shengyuan $X u^{2}$, Eric Rogers ${ }^{3}$, D. H. Owens ${ }^{4}$ \\ ${ }^{1}$ Institute of Control and Computation Engineering, \\ University of Zielona Góra, Poland. \\ ${ }^{2}$ Department of Mechanical Engineering, \\ University of Hong Kong, China. \\ ${ }^{3}$ Department of Electronics and Computer Science, \\ University of Southampton, UK. \\ ${ }^{4}$ Department of Automatic Control and Systems Engineering, \\ University of Sheffield, UK.
}

\begin{abstract}
In this paper, we study the stability and the stabilisation of $2 D$ discrete linear systems with multiple state delays. All of the new results obtained are based on analysis of the Fornasini-Marchesini state space model with delays and the resulting conditions are given in terms of linear matrix inequalities (LMIs). A numerical example is given to illustrate the effectiveness of the overall approach.
\end{abstract}

\section{INTRODUCTION}

The analysis of time-delay systems is a very important part of (linear) systems theory and has been a very active research area over the past few decades. The interest in time-delay systems stems from the fact that such delays occur often in, for example, electronic, mechanical, biological, metallurgical and chemical systems - see, for example, $[10,11]$.

The existence of delays is frequently a source of instability. Much work has been reported on the problem of the stability of standard, termed $1 D$ here, linear systems with delays $[4,12]$ but relatively little on the stability of $2 D(n D)$ linear systems with delays.

In this paper, we develop stability conditions for $2 D$ linear systems with multiple state delays and then establish some connections between multidimensional delay and delay-free systems. Based on the these results conditions for the existence of stabilising controllers are developed. All of these conditions are formulated in terms of linear matrix inequalities (LMIs) $[2,6]$, where an advantage of using LMIs is the fact that there exist efficient numerical algorithms to solve them as demonstrated by the numerical example which concludes this paper.

Throughout this paper, the null matrix and the identity matrix with appropriate dimensions are denoted by 0 and $I$ respectively. $M>0$ is used to denote the fact that $M$ is a real symmetric positive definite matrix. Also, delays $h_{1} \cdots, h_{q}$ are termed noncommensurate if $\exists$ no integers $l_{1}, \cdots, l_{q}$ (not all of them zero) such that $\sum_{i=1}^{q} l_{i} h_{i}=0$. The underlying delay differential system is ter- med commensurate if $q=1$. We will also make extensive use of the following well known result.

Lemma 1 (Schur complement) [2] For matrices $\Sigma_{1}, \Sigma_{2}$, and $\Sigma_{3}$ where $\Sigma_{1}>0$ and $\Sigma_{3}=\Sigma_{3}^{T}$ then

$$
\Sigma_{3}+\Sigma_{2}^{T} \Sigma_{1}^{-1} \Sigma_{2}<0
$$

if, and only if.

$$
\left[\begin{array}{cc}
\Sigma_{3} & \Sigma_{2}^{T} \\
\Sigma_{2} & -\Sigma_{1}
\end{array}\right]<0 \text { or }\left[\begin{array}{cc}
-\Sigma_{1} & \Sigma_{2} \\
\Sigma_{2}^{T} & \Sigma_{3}
\end{array}\right]<0
$$

\section{2. $2 D$ LINEAR SYSTEMS WITH MULTIPLE DELAYS}

Consider a $2 D$ linear system with multiple state delays which can be represented by the Fornasini-Marchesini state-space model [5] with delays

$$
\begin{aligned}
x(i+1, j+1)= & A_{1} x(i+1, j)+A_{2} x(i, j+1) \\
& +\sum_{k=1}^{s_{1}} A_{1 k d} x\left(i+1, j-d_{1 k}\right) \\
& +\sum_{l=1}^{s_{2}} A_{2 l d} x\left(i-d_{2 l}, j+1\right) \\
& +B_{1} u(i+1, j)+B_{2} u(i, j+1) \\
& +\sum_{k=1}^{s_{1}} B_{1 k d} u\left(i+1, j-d_{1 k}\right) \\
& +\sum_{i=1}^{s_{2}} B_{2 l d} u\left(i-d_{2 l}, j+1\right)
\end{aligned}
$$

where $x(i, j) \in R^{n}, u(i, j) \in R^{m}$ are the state and input vectors respectively, $i, j \in Z_{+}$, where $Z_{+}$denotes the set of nonnegative integers, $A_{p}, B_{p}(p=1,2), A_{1 k d}, B_{1 k d} k=1, \ldots, s_{1}, A_{2 l d}$, $B_{2 l d} l=1, \ldots, s_{2}$ are known constant matrices with compatible dimensions, and $s_{1}$ and $s_{2}$ denote the number of delay terms in each direction respectively. We also assume that $0<d_{11}<d_{12}<$ 
$\cdots<d_{1 s_{1}}$ and $0<d_{21}<d_{22}<\cdots<d_{2 s_{2}}$ and in this case the boundary conditions are defined as

$$
\begin{aligned}
& \left\{x(i, j)=v_{i j}\right\}, \forall i \geq 0 ; j=-d_{1 s_{1}},-d_{1 s_{1}}+1, \ldots, 0 \\
& \left\{x(i, j)=w_{i j}\right\}, \forall j \geq 0 ; i=-d_{2 s_{2}},-d_{2 s_{2}}+1, \ldots, 0
\end{aligned}
$$

where $v_{00}=w_{00}$.

With $X_{r}=\sup \{\|x(i, j)\|: i+j=r, i, j \in \mathbb{Z}\}$, asymptotic stability of the model ( 3 ) is defined as follows.

Definition $1[8,5]$ The $2 D$ linear system with multiple state delays (3) is said to be asymptotically stable if

$\lim X_{r}=0$ for zero input $u(i, j)=0$ and for any bounded boundary conditions of the form (4).

\subsection{Noncommensurate delays}

In the case of noncommensurate delays, the following result characterizes asymptotic stability of the class of systems considered in terms of an LMI condition.

Theorem 1 The $2 D$ delay system ( 3 ) is asymptotically stable if $\exists$ matrices $P, Q>0, U_{11}, \ldots, U_{1 s_{1}}>0$ and $U_{21}$, $\ldots, U_{2 s_{2}}>0$ such that the following $L M I$ holds:

$$
\begin{aligned}
& {\left[\begin{array}{c}
A_{1}^{T} \\
A_{2}^{T} \\
\Lambda_{1 d}^{T} \\
\Lambda_{2 d}^{T}
\end{array}\right] P\left[\begin{array}{lllll}
A_{1} & A_{2} & \Lambda_{1 d} & \Lambda_{2 d}
\end{array}\right]} \\
& -\left[\begin{array}{cccc}
P-Q-\Phi_{1}-\Phi_{2} & 0 & 0 & 0 \\
0 & Q & 0 & 0 \\
0 & 0 & \Omega_{1} & 0 \\
0 & 0 & 0 & \Omega_{2}
\end{array}\right]<0
\end{aligned}
$$

where

$$
\begin{aligned}
& \Lambda_{1 d}=\left[A_{11 d}, A_{12 d}, \ldots, A_{1 s_{1} d}\right], \Phi_{1}=\sum_{k=1}^{s_{1}} Q_{1 k} \\
& \Lambda_{2 d}=\left[A_{21 d}, A_{22 d}, \ldots, A_{2 s_{2} d}\right], \Phi_{2}=\sum_{l=1}^{s_{2}} Q_{2 l} \\
& \Omega_{1}=\operatorname{diag}\left(Q_{11}, Q_{12}, \ldots, Q_{1 s_{1}}\right), Q_{1 k}=\sum_{\theta=1}^{s_{1}-k+1} U_{1 \theta} \\
& \Omega_{2}=\operatorname{diag}\left(Q_{21}, Q_{22}, \ldots, Q_{2 s_{2}}\right), Q_{2 l}=\sum_{\theta=1}^{s_{2}-l+1} U_{2 \theta}
\end{aligned}
$$

Proof: This is via a Lyapunov-Krasovskii approach. In particular, suppose that $V(\zeta, \xi)$ denotes a function that expresses the energy stored at $x(i+\zeta, j+\xi)$ and consider the particular case when

$$
V(\zeta, \xi)=x^{T}(i+\zeta, j+\xi) W_{\zeta \xi} x(i+\zeta, j+\xi)
$$

where $W_{\zeta \xi}>0$ is given and $\zeta, \xi \in Z_{+}, \zeta \geq-d_{1 s_{1}}, \xi \geq-d_{2 s_{2}}$. Now introduce the following candidate Lyapunov functions for the delayed terms:

$$
\begin{aligned}
V_{d_{1}}(\zeta, \xi)= & x^{T}(i+\zeta, j+\xi) W_{\zeta \xi} x(i+\zeta, j+\xi) \\
& +\sum_{k=1}^{s_{1}} \sum_{\theta=-d_{1 k}}^{-1} x^{T}(i+\zeta, j+\theta) U_{1 k} x(i+\zeta, j+\theta) \\
V_{d_{2}}(\zeta, \xi)= & x^{T}(i+\zeta, j+\xi) W_{\zeta \xi} x(i+\zeta, j+\xi) \\
& +\sum_{l=1}^{s_{2}} \sum_{\theta=-d_{2 l}}^{-1} x^{T}(i+\theta, j+\xi) U_{2 l} x(i+\theta, j+\xi)
\end{aligned}
$$

where $W_{\zeta \zeta}>0$ and $U_{1 k}, U_{2 l}>0$ are given and $\zeta, \xi \in Z, \zeta \geq$ $-d_{1 s_{1}}, \xi \geq-d_{2 s_{2}}$. In order to determine the change of the energy in the both sides of (3) consider the increment $\Delta V(i, j)$ where

$$
\Delta V(i, j)=V_{1,1}(i, j)-V_{d_{1}}(1,0)-V_{d_{2}}(0,1)
$$

Now consider the result of substituting (7) and (8) into (9) and define the augmented state vector as

$$
\begin{aligned}
\hat{x}= & {\left[x^{T}(i+1, j) x^{T}(i, j+1) x^{T}\left(i+1, j-d_{11}\right)\right.} \\
& x^{T}\left(i+1, j-d_{12}\right) \ldots x^{T}\left(i+1, j-d_{1 s_{1}}\right) \\
& x^{T}\left(i-d_{21}, j+1\right) x^{T}\left(i-d_{22}, j+1\right) \ldots \\
& \left.x^{T}\left(i-d_{2 d_{s_{2}}}, j+1\right) \quad x_{l}^{T} \quad x_{k}^{T}\right]
\end{aligned}
$$

where $x_{l}$ includes all states from $x(i+1, j-1) \ldots x(i+1, j-$ $\left.d_{1 s_{1}}+1\right)$ excluding those defined before and $x_{l}$ includes all states from $x(i-1, j+1) \ldots x\left(i-d_{2 s_{2}}+1, j+1\right)$ but also excluding those defined before. Then (9) can be rewritten as (using the same notation as in (6))

$$
\Delta V(i, j)=\widehat{x}^{T} \Pi \widehat{x}=\hat{x}^{T}\left(\Theta^{T} W_{11} \Theta-\Xi\right) \hat{x}
$$

where

$$
\begin{aligned}
& \Theta=\left[\begin{array}{llllll}
A_{1} & A_{2} & \Lambda_{1 d} & \Lambda_{2 d} & 0 & 0
\end{array}\right], \\
& \Xi=\operatorname{diag}\left(W_{10}, W_{01}, \Omega_{1}, \Omega_{2}, \Omega_{3}, \Omega_{4}\right)
\end{aligned}
$$

Now, if $\Delta V(i, j)<0$ for $\hat{x} \neq 0$, then the 2D discrete linear system considered here is asymptotically stable. In order to guarantee that this stability condition holds, it is clear that $\Pi<0$ must hold. Also the last two rows and columns in this matrix (i.e. those which only consist of $-\Omega_{3}$ and $-\Omega_{4}$ ) in (10) can be omitted because they only contribute terms that are guaranteed to be negative definite. By again making use of $(7)$ and $(8)$ it is easily seen that to guarantee the dissipative property $(\Delta V(i, j)<0)$ we can choose

$$
\begin{array}{r}
W_{11}=P, Q_{1 k}=\sum_{\theta=1}^{s_{1}-k+1} U_{1 \theta}, Q_{2 l}=\sum_{\theta=1}^{s_{2}-l+1} U_{2 \theta}, W_{01}=Q \\
W_{10}=P-Q-Q_{11}-\ldots-Q_{1 k}-Q_{21}-\ldots-Q_{2 l},
\end{array}
$$

Remark 1 It was shown in [9] (see also [3] and [1]) that there exist connections between (linear) $2 D$ delay-free systems and $1 D$ time-delay systems. These arise because the delayed signal in the $1 D$ case can be viewed as a signal transmitted through another dimension in the $2 D$ framework. Theorem 1 here shows that the same result can be established for $2 D$ linear systems with multiple delays. Hence, asymptotic stability of a $2 D$ linear system with $m_{1}$ and $m_{2}$ delayed terms in each direction respectively is equivalent to asymptotic stability of an $m D$ linear system where $m=m_{1}+$ $m_{2}+2$. 


\subsection{Commensurate Delays}

In what follows, we show that if all delays present in (5) are commensurate then investigation of the stability properties of $2 D$ delay system can be equivalently treated as the stability investigation of a $4 D$ delay free system. The key to establishing this fact is the Elementary Operation Algorithm (EOA) developed by Gałkowski [7].

In general case, the notation associated with this area is very cumbersome and hence for ease of presentation only we consider the particular case of a $2 D$ linear system of the form (3) with two delays in each direction, i.e. we restrict attention to $m_{1}=m_{2}=$ 2. In which case it is clear that the associated characteristic polynomial for stability is given by the determinant of the following 2D polynomial matrix

$$
\begin{aligned}
& I-A_{1} z_{1}^{-1}-A_{2} z_{2}^{-1}-A_{3} z_{1}^{-h_{1} k}-A_{4} z_{1}^{-h_{2} k} \\
& -A_{5} z_{2}^{-p_{1} l}-A_{6} z_{1}^{-p_{2} l}
\end{aligned}
$$

where $k, l \in R^{+}$and $h_{1}, h_{2}, p_{1}, p_{2}$ are natural numbers. Now introduce the new variables $z_{1}^{k}=z_{3}, z_{2}^{l}=z_{4}$ and then rewrite (12) as

$$
\begin{aligned}
& I-A_{1} z_{1}^{-1}-A_{2} z_{2}^{-1}-A_{3} z_{3}^{-h_{1}}-A_{4} z_{3}^{-h_{2}} \\
& -A_{5} z_{4}^{-p_{1}}-A_{6} z_{4}^{-p_{2}} .
\end{aligned}
$$

Assume also that $h_{1}=1, h_{2}=2, p_{1}=1, p_{2}=2$ which yields

$$
\begin{aligned}
& I-A_{1} z_{1}^{-1}-A_{2} z_{2}^{-i}-A_{3} z_{3}^{-1}-A_{4} z_{3}^{-2} \\
& -A_{5} z_{4}^{-1}-A_{6} z_{4}^{-2} .
\end{aligned}
$$

Application of the EOA to this last $4 D$ polynomial matrix now gives

$$
\left[\begin{array}{ccc}
I & 0 & z_{4}^{-1} A_{5} \\
0 & I & z_{3}^{-1} A_{4} \\
z_{4}^{-1} & z_{3}^{-1} & I-A_{1} z_{1}^{-1}-A_{2} z_{2}^{-1}-A_{3} z_{3}^{-1}-A_{6} z_{4}^{-1}
\end{array}\right]
$$

which is equivalent to

$$
I-\widehat{A}_{1} z_{1}^{-1}-\widehat{A}_{2} z_{2}^{-1}-\widehat{A}_{3} z_{3}^{-1}-\widehat{A}_{4} z_{4}^{-1}
$$

where

$$
\begin{gathered}
\widehat{A}_{1}=\left[\begin{array}{ccc}
0 & 0 & 0 \\
0 & 0 & 0 \\
0 & 0 & A_{1}
\end{array}\right], \widehat{A}_{2}=\left[\begin{array}{ccc}
0 & 0 & 0 \\
0 & 0 & 0 \\
0 & 0 & A_{2}
\end{array}\right], \\
\widehat{A}_{3}=\left[\begin{array}{ccc}
0 & 0 & 0 \\
0 & 0 & -A_{4} \\
0 & -I & A_{3}
\end{array}\right], \widehat{A}_{4}=\left[\begin{array}{ccc}
0 & 0 & -A_{5} \\
0 & 0 & 0 \\
-I & 0 & A_{6}
\end{array}\right]
\end{gathered}
$$

Here only elementary operations that preserve the matrix determinant are used and hence it is straightforward to see that (13) and (15) have the same determinant and hence the stability property for both system descriptions is the same. This result is easily extended to the partially commensurate case. In particular, assume that each delay $d_{1 v}, v=1, \ldots, m_{1}$ is a multiple of one of basic noncommensurate delays $k_{1}, k_{2}, \ldots, k_{t_{1}}$ and similarly for $d_{1 h}, h=1, \ldots, m_{2}$ of $l_{1}, l_{2}, \ldots, l_{t_{2}}$. Then the previous method exploiting this fact requires the investigation of an $n D$ linear system, where $n=t_{1}+t_{2}+2$ whereas the method of Theorem 1 here requires the investigation of an $m D$ linear system with $m=m_{1}+m_{2}+2$.

\section{STABILIZATION 2D LINEAR SYSTEM WITH MULTIPLE DELAYS}

Consider a 2D linear system with multiple state and input delays described by (3) and assume that the state feedback control law

$$
u(i, j)=K x(i, j)
$$

is used. The corresponding closed-loop system is

$$
\begin{aligned}
x(i+1, j+1)= & \left(A_{1}+B_{1} K\right) x(i+1, j) \\
& +\left(A_{2}+B_{2} K\right) x(i, j+1) \\
& +\sum_{k=1}^{s_{1}}\left(A_{1 k d}+B_{1 k d} K\right) x\left(i+1, j-d_{1 k}\right) \\
& +\sum_{i=1}^{s_{2}}\left(A_{2 l d}+B_{2 l d} K\right) x\left(i-d_{2 l}, j+1\right)
\end{aligned}
$$

Definition 2 If there exists $K$ such that (17) is asymptotically stable, then the $2 D$ delay system (3) is said to be stabilisable.

Theorem 2 The $2 D$ delay system (3) is asymptotically stable if $\exists$ matrices $W, Z>0, Z_{11}, \ldots, Z_{1 s_{1}}>0$,

$Z_{21}, \ldots, Z_{2 s_{2}}>0$ and any $N$ such that the following LMI holds:

$$
\begin{gathered}
{\left[\begin{array}{ccc}
-W & A_{1} W+B_{1} N \\
W A_{1}^{T}+N^{T} B_{1}^{T} & -\Upsilon \\
W A_{2}^{T}+N^{T} B_{2}^{T} & 0 \\
\Upsilon_{1 d}^{T} & & 0 \\
\Upsilon_{2 d}^{T} & & 0 \\
A_{2} W+B_{2} N & \Upsilon_{1 d} & \Upsilon_{2 d} \\
0 & 0 & 0 \\
-Z & 0 & 0 \\
0 & -Z_{1 d} & 0 \\
0 & 0 & -Z_{2 d}
\end{array}\right]<0}
\end{gathered}
$$

where

$$
\begin{aligned}
& \Upsilon=W-Z-R_{11}-\ldots-R_{1 s_{1}}-R_{21}-\ldots-R_{2 s_{2}} \\
& \Upsilon_{1 d}=\left[A_{11 d} W+B_{11 d} N, \quad A_{12 d} W+B_{12 d} N,\right. \\
& \left.\ldots, A_{1 s_{1} d}+B_{1 s_{1} d} N\right] \text {, } \\
& \Upsilon_{2 d}=\left[A_{21 d} W+B_{21 d} N, \cdot A_{22 d} W+B_{22 d} N,\right. \\
& \left.\ldots, A_{2 s_{2} d}+B_{2 s_{2} d} N\right] \text {, } \\
& Z_{1 d}=\operatorname{diag}\left(R_{11}, R_{12}, \ldots, R_{1 s_{1}}\right) \text {, } \\
& Z_{2 d}=\operatorname{diag}\left(R_{21}, R_{22}, \ldots, R_{2 s_{2}}\right) \text {, } \\
& Z=W Q W, R_{1 k}=\sum_{\theta=1}^{s_{1}-k+1} Z_{1 \theta}=\sum_{\theta=1}^{s_{1}-k+1} W U_{1 \theta} W, \\
& R_{2 l}=\sum_{\theta=1}^{s_{2}-l+1} Z_{2 \theta}=\sum_{\theta=1}^{s_{2}-l+1} W U_{2 \theta} W,
\end{aligned}
$$

If this condition holds, then the system is stabilised by feedback of $K=N W^{-1}$

Proof: Using (5) and (16) and applying the Shur's complement (2), the closed-loop system is asymptotically stable if $\exists$ matrices 
$P, Q>0, U_{11}, \ldots, U_{1 s_{1}}>0$ and $U_{21} \ldots$,

$U_{2 s_{2}}>0$ such that

$$
\begin{gathered}
{\left[\begin{array}{ccc}
-P & P A_{1}+P B_{1} K \\
A_{1}^{T} P+K^{T} B_{1}^{T} P & -\Gamma \\
A_{2}^{T} P+K^{T} B_{2}^{T} P & 0 \\
\Gamma_{1 d}^{T} & & 0 \\
\Gamma_{2 d}^{T} & & 0 \\
P A_{2}+P B_{2} K & \Gamma_{1 d} & \Gamma_{2 d} \\
0 & 0 & 0 \\
-Q & 0 & 0 \\
0 & -\Omega_{1} & 0 \\
0 & 0 & -\Omega_{2}
\end{array}\right]<0}
\end{gathered}
$$

where

$$
\begin{aligned}
\Gamma= & P-Q-\Phi_{1}-\Phi_{2} \\
\Gamma_{1 d}= & {\left[P A_{11 d}+P B_{11 d} K, \quad P A_{12 d}+P B_{12 d} K,\right.} \\
& \left.\ldots, P A_{1 s_{1 d}}+P B_{1 s_{1} d} K\right], \\
\Gamma_{2 d}= & {\left[P A_{21 d}+P B_{21 d} K, P A_{22 d}+P B_{22 d} K,\right.} \\
& \left.\ldots, P A_{2 s_{2} d}+P B_{2 s_{2} d} K\right]
\end{aligned}
$$

Now set $P=W^{-1}$ and apply the congruence transformation defined by $\operatorname{diag}(W, W, \ldots, W)$. Then employ the notation (19) to obtain (18)

\section{A NUMERICAL EXAMPLE}

We illustrate the results developed in this paper via one example where the computations involved have been undertaken using the LMI Control Toolbox [6].

Consider the following 2D system of the type (3) with 4 delays (for simplicity, we assume 2 delays in each direction) described by

$$
\begin{aligned}
& A_{1}=\left[\begin{array}{ll}
0.2 & 0.1 \\
0.4 & 0.9
\end{array}\right], A_{2}=\left[\begin{array}{ll}
0.4 & 0.5 \\
0.4 & 0.3
\end{array}\right], B_{1}=\left[\begin{array}{ll}
0.5 & 0.5 \\
0.3 & 0.8
\end{array}\right], \\
& B_{2}=\left[\begin{array}{ll}
0.5 & 0.2 \\
0.6 & 0.3
\end{array}\right], A_{11 d}=\left[\begin{array}{ll}
0.7 & 0.4 \\
0.6 & 0.5
\end{array}\right], A_{12 d}=\left[\begin{array}{ll}
0.7 & 0.6 \\
0.1 & 0.1
\end{array}\right], \\
& A_{21 d}=\left[\begin{array}{ll}
0.4 & 0.9 \\
0 & 0.1
\end{array}\right], A_{22 d}=\left[\begin{array}{cc}
0 & 1.0 \\
0.9 & 0.7
\end{array}\right], B_{11 d}=\left[\begin{array}{ll}
0.4 & 0.2 \\
0.3 & 0.4
\end{array}\right], \\
& B_{12 d}=\left[\begin{array}{ll}
0.3 & 0.7 \\
0.2 & 0.8
\end{array}\right], B_{21 d}=\left[\begin{array}{ll}
0.7 & 0.4 \\
0.2 & 0.8
\end{array}\right], B_{22 d}=\left[\begin{array}{ll}
0.6 & 0.8 \\
0.2 & 0.8
\end{array}\right]
\end{aligned}
$$

Note that this particular example is unstable. Also the following matrices solve the LMI condition (18) in this case

$$
\begin{aligned}
& W=\left[\begin{array}{cc}
1.7828 & -0.3422 \\
-0.3422 & 2.1173
\end{array}\right], Z=\left[\begin{array}{cc}
3.9047 & -0.0398 \\
-0.0398 & 3.9436
\end{array}\right], \\
& Z_{11}=\left[\begin{array}{ll}
2.3288 & 0.0159 \\
0.0159 & 2.3133
\end{array}\right], Z_{12}=\left[\begin{array}{ll}
3.0958 & 0.0478 \\
0.0478 & 3.0491
\end{array}\right] \text {, } \\
& Z_{21}=\left[\begin{array}{ll}
2.3288 & 0.0159 \\
0.0159 & 2.3133
\end{array}\right], Z_{22}=\left[\begin{array}{ll}
3.0958 & 0.0478 \\
0.0478 & 3.0491
\end{array}\right] \\
& \text { and } N=\left[\begin{array}{ll}
-0.6351 & -1.4445 \\
-0.3656 & -0.7135
\end{array}\right]
\end{aligned}
$$

Hence the system (4) is asymptotically stable independent of the delays under the control law (16) with

$$
K=\left[\begin{array}{cc}
-0.5028 & -0.7635 \\
-0.2784 & -0.3820
\end{array}\right]
$$

\section{CONCLUSIONS}

This paper has considered particular aspects of the stability of $2 D$ linear systems with multiple state delays. All new results are expressed in terms of LMIs and hence their actual implementation for numerical examples can, in principle, follow immediately. One potential problem with this approach, however, is the fact that the dimensions of the matrices involved in the LMI based conditions could well be very large and hence numerical difficulties could arise. This can occur, for example, when the system dimensionality is large $(n \gg 1)$ and/or many delays are present. This aspect is clearly one to which further research effort could be applied. Another key feature of the stability tests in this paper is that they extend in a natural manner to the design of stabilizing control laws - a feature which is not available for other stability tests in the many particular cases of processes with $2 D / n D$ linear dynamics.

\section{REFERENCES}

[1] P. Agathoklis and S. Foda. Stability and the matrix Lyapunov equation for delay differential systems. International Journal of Control, 49(2):417-432, 1989.

[2] S. Boyd, L. E. Ghaoui, E. Feron, and V. Balakrishnan. Linear Matrix Inequalities In System And Control Theory, volume 15 of SIAM studies in applied mathematics. SIAM. Philadelphia, 1994.

[3] J. N. Chiasson, S. D. Brierley, and E.B. Lee. A simplified derivation of the Zeheb-Walch 2-D stability test with applications to time-delay systems. IEEE Trans. Automat. Control, AC-30(4):411-414, 1985.

[4] L. Dugard and E. I. Verriest. Stability and Control of Timedelay Systems, volume 228 of $L N C I S$. Springer-Verlag, London, 1998.

[5] E. Fomasini and G. Marchesini. Doubly indexed dynamical systems ; state-space models and structural properities. Mathematical System Theory, 1(1), 1978.

[6] P. Gahinet, A. Nemirowski, A. J. Laub, and M. Chilali. LMI Control Toolbox for use with MATLAB. The Mathworks Partner Series. The MathWorks Inc., 1995.

[7] K. Gałkowski. State-Space Realisations of Linear 2-D Systems with Extensions to the General nD Case, volume 263 of LNCIS. Springer Verlag, London, 2001.

[8] T. Kaczorek. Two-dimensional Linear Systems, volume 68 of $L N C I S$. Springer-Verlag, Berlin, 1985.

[9] E. W. Kamen. On the relationship between zero criteria for two-variable polynomials and asymptotic stability of delay differential equations. IEEE Trans. Automat. Control, AC23:983, 1980.

[10] M. S. Mahmoud. Robust Control and Filtering For TimeDelay Systems, volume 5 of Control Engineering Series. Marcel Dekker, Inc., 2000.

[11] M. Malek-Zavarei and M. Jamshidi. Time-Delay Systems: Analysis, Optimization and Aplications, volume 9 of $\mathrm{North}$ Holland Systems and Control. North-Holland, 1987.

[12] S.-I. Niculescu. Delay Effects on Stability, volume 269 of LNCIS. Springer Verlag, London, 2001. 